\title{
Erik W. Grafarend and Joseph L. Awange: Applications of Linear and Nonlinear Models - Fixed Effects, Random Effects, and Total Least Squares*
} Book Review

Lars E. Sjöberg

This very comprehensive encyclopaedia on linear and nonlinear algebra altogether contains more than 1000 pages, including 115 pages of some 3000 references. The senior author is eminent in the field of geodetic adjustment with at least two previous text books on linear models, and the two author already have a long co-operation in writing text books. Hence, the appearance of a new book by them in this subject area is a notable event for everyone seriously interested in algebra and treatment of systems of equations with or without real data. The book should be a rich source of information and inspiration for anyone interested in solving systems of linear and nonlinear equations of all kinds. The readership includes scholars in mathematics, experimental physics, natural sciences, geodesy, engineering and economy. As can be seen from Table 1, the contents of the book is well structured, dealing with a large variety of items and with a unique distinction between algebraic and probabilistic regressions, the latter solutions attached with a thorough description of methods for variance-covariance estimation and confidence intervals. Nonlinear problems are either treated by standard Taylor series linearization or, sometimes, by special closed-form techniques.
The appendices are condensed descriptions of various algebras, sampling distributions. Each chapter has a notable introduction as a "manual" to any reader. The mathematical details are carefully described in the Appendices, which should be of particular interest to geodesists. All over the book the problems and their solutions are generously illustrated with figures and instructive numerical examples. Therefore, it could also be recommended for graduate students, but for many of them some missing basic definitions (such as tensor product and matrix properties reflexivity, idempotency, etc.) must be searched elsewhere, and the many acronyms could have been explained in an introductory list, and also I found one computational error in a numerical example. Nevertheless, I admire the authors' rigour and clear descriptions of all chapters. The book is a must for each library on the above cited sciences, and only the price makes me hesitate from recommending it for the personal libraries.

Lars E. Sjöberg 
Table 1. Structures of chapters for solving various systems of equations.

Abbreviations: MINOS $=$ Minimum Norm Solution, LUMBE $=$ Linear Uniformly Minimum Bias Estimator, LESS $=$ Least Squares Solution MINOLESS $=$ Minimum Norm Least Squares Solution, GGM = Gauss Markov Model, BLUUE $=$ Best Linear Uniformly Unbiased Estimator, BIQUUE = Best Invariant Quadratic Uniformly Unbiased Estimator, BLUMBE = Best Linear Minimum Bias Estimator

\begin{tabular}{lll}
\hline Type of system of equations & Algebraic problems & Probabilistic problems \\
\hline \hline Consistent & MINOS & LUMBE \\
Inconsistent with no datum defect & LESS & GMM without Datum Defect BLUUE and BIQUUE \\
Inconsistent with datum effect & MINOLESS & GMM with Datum Defect BLUMBE \\
\hline
\end{tabular}

Special chapters:

- Nonlinear problems of 3D datum equations

- Gauss-Helmert Model

- Special problems of Algebraic Regression and Stochastic Estimation

- Algebraic solutions of Linear and nonlinear equations

Appendices:

- Tensor, Linear, Matrix and Multilinear Algebras

- Sampling Distributions and their use

- Statistical notations, random events and stochastic processes

- Basics of Groebner Basis Algebra 